Supplement of Earth Syst. Dynam., 10, 189-203, 2019

https://doi.org/10.5194/esd-10-189-2019-supplement

(C) Author(s) 2019. This work is distributed under

the Creative Commons Attribution 4.0 License.

(c) (1)
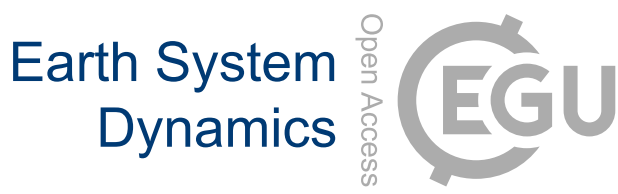

Supplement of

\title{
September Arctic sea ice minimum prediction - a skillful new statistical approach
}

Monica Ionita et al.

Correspondence to: Monica Ionita (monica.ionita@awi.de)

The copyright of individual parts of the supplement might differ from the CC BY 4.0 License. 


\section{Workflow for the selection of the stability maps and the optimal forecast model.}

1. SSIE is correlated with numerous potential predictors (e.g. OHC, OT100, SST, SLP, TT, PWC, DW, USURF and VSURF). Different lags (Table2) and regions of the same variable are regarded as independent predictor.

2. The correlation is computed in a moving window of 21 years within the period from 1979 to 2007 (window 1979-2000, window 2: 1980-2001, .., window 1986-2007).

3. The correlation is considered stable for those grid-points where SSIE and the large-scale predictors are significantly correlated at $95 \%, 90 \%, 85 \%$ and $80 \%$ significance level for more than $80 \%$ of the 21 -year windows, covering the period 1979-2007. The area where the correlation coefficient is stable and positive are represented as dark red $(95 \%)$, red $(90 \%)$, orange $(85 \%)$ and yellow $(80 \%)$, while the regions where correlation coefficient is stable and negative are represented as dark blue (95\%), blue (90\%), green (85\%) and light green $(80 \%)$. Such maps are referred in our study as stability maps.

4. The optimal predictors are defined as the average values over the stable regions for each gridded parameter (Figures S3 - S15).

5. Only regions where the correlation is above $90 \%$ significance level, are retained for further analysis.

6. The final composition of the predictors (Figure $1-3$ and $5-7$ ) for the forecasting model is established by stepwise regression of the stable predictors using the Akaike information criterion and the explained variance of forecast errors.

\section{Skill measures}

To better assess the skill of the forecast, different statistical metrics have been employed: mean absolute error (MAE), mean square error (MSE), root mean square error (RMSE), normalized root mean square error (NMRSE), Nush - Sutcliffe Efficency (NSE), modified NSE (mNSE), relative NSE (rNSE), index of agreement (d), coefficient of persistence $(\mathrm{CP})$ and coefficient of determination $\left(\mathrm{R}^{2}\right)$.

1. Mean absolute error (MAE)

$$
m a e=\frac{1}{N} \sum_{i=1}^{N}\left|\left(S_{i}-O_{i}\right)\right|
$$

2. Mean square error (MSE)

$$
m s e=\frac{1}{N} \sum_{i=1}^{N}\left(S_{i}-O_{i}\right)^{2}
$$

3. Root mean square error (RMSE)

$$
r m s e=\sqrt{\frac{1}{N} \sum_{i=1}^{N}\left(S_{i}-O_{i)}{ }^{2}\right.}
$$

4. Normalized root mean square error (NRMSE)

$$
\begin{aligned}
& n r m s e=100 \frac{\sqrt{\frac{1}{N} \sum_{i=1}^{N}\left(S_{i}-O_{i}\right)^{2}}}{n v a l} \\
& \text { where } \quad n v a l=\left\{\begin{array}{l}
s d\left(O_{i}\right), \quad \text { norm }=" s d " \\
O_{\text {max }}-O_{\text {min }},
\end{array} \text { norm }=\right.\text { "maxim" }
\end{aligned}
$$




$$
N S E=1-\frac{\sum_{i=1}^{N}\left(S_{i}-O_{i}\right)^{2}}{\sum_{i=1}^{N}\left(O_{i}-\bar{O}\right)^{2}}
$$

NSE (Nush and Sutcliffe, 1970) ranges from -Inf to 1. Essentially, the closer to 1, the more accurate the model is. $\mathrm{NSE}=1$ indicates a prefect forecast model, NSE $=0$ indicates that the model predictions are as accurate as the mean of the observed data and $-\mathrm{Inf}<\mathrm{NSE}<0$, indicates that the observed mean is better predictor than the model.

6. Modified NSE (mNSE)

$$
m N S E=1-\frac{\sum_{i=1}^{N}\left|S_{i}-O_{i}\right|^{j}}{\sum_{i=1}^{N}\left|O_{i}-\bar{O}\right|^{j}}
$$

\section{Relative NSE (rNSE)}

$$
r N S E=1-\frac{\sum_{i=1}^{N}\left(\frac{S_{i}-O_{i}}{\bar{O}}\right)^{2}}{\sum_{i=1}^{N}\left(\frac{O_{i}-\bar{O}}{\bar{O}}\right)^{2}}
$$

8. Index of Agreement $(0<=\mathrm{d}<=1)$

$$
d=1-\frac{\sum_{i=1}^{N}\left(O_{i}-S_{i}\right)^{2}}{\sum_{i=1}^{N}\left(\left|S_{i}-\bar{O}\right|+\left|O_{i}-\bar{O}\right|\right)^{2}}
$$

The Index of Agreement (d) developed by Willmot (1982) as a standardized measure of the degree of model prediction errors and varies between 0 and 1 . A value of 1 indicates a perfect match and 0 indicates no agreement at all.

9. Coefficient of persistence $(0<=\mathrm{CP}<=1)$.

$$
C P=1-\frac{\sum_{i=2}^{N}\left(S_{i}-O_{i}\right)^{2}}{\sum_{i=1}^{N-1}\left(O_{i+1}-O_{i}\right)^{2}}
$$

The coefficient of persistence compares the predictions of the model with the predictions obtained by assuming that the process is a Wiener process (variance increasing linearly with time), in which case, the best estimate for the future is given by the latest measurement (Kitanidis and Bras, 1980). Persistence model efficiency is a normalized model evaluation statistic that quantifies the relative magnitude of the residual variance (noise) to the variance of the errors obtained by the use of a single persistence model (Moriasi et al., 2007). The coefficient of persistence ranges from 0 to 1 , with $\mathrm{CP}=1$ being the optimal value and it should be larger than 0 to indicate a minimally acceptable performance model.

\section{References}

Kitanidis, P.K., and Bras, R.L. 1980. Real-time forecasting with a conceptual hydrologic model. 2. Applications and results. Water Resources Research, Vol. 16, No. 6, pp. 1034:1044.

Moriasi, D. N. et al. (2007). Model Evaluation Guidelines for Systematic Quantification of Accuracy in Watershed Simulations. Transactions of the ASABE, 50:(3), 885-900.

Nash, J. E. and Sutcliffe, J. V. 1970: River flow forecasting through conceptual models, Part I - A discussion of principles, J. Hydrol., 10,282-290.

Willmot, C. J. 1981: On the validation of models, Physical Geography, 2,184-194. 
a)

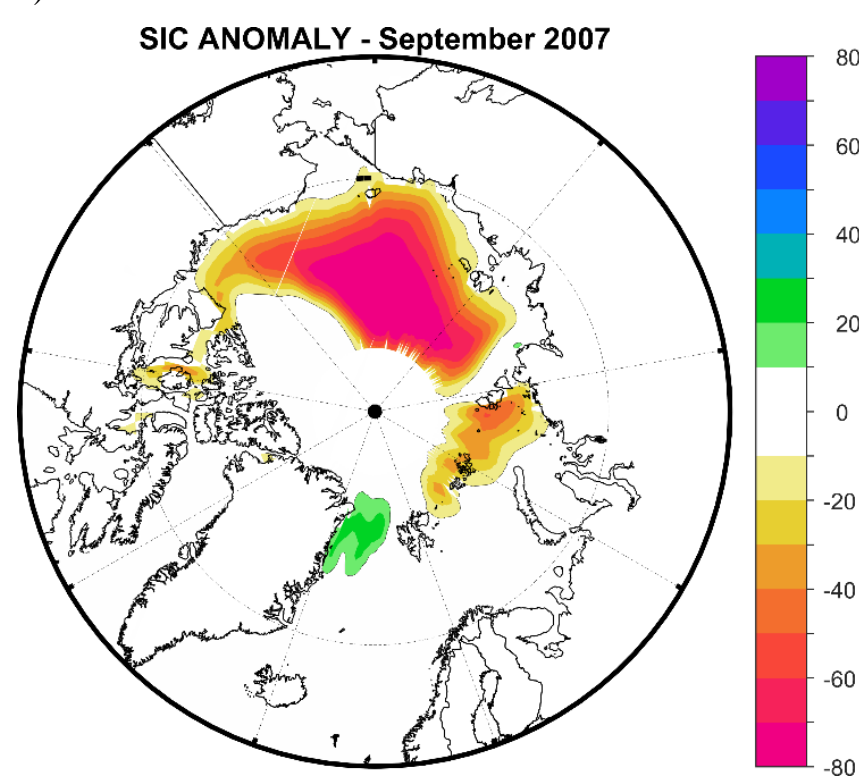

b)

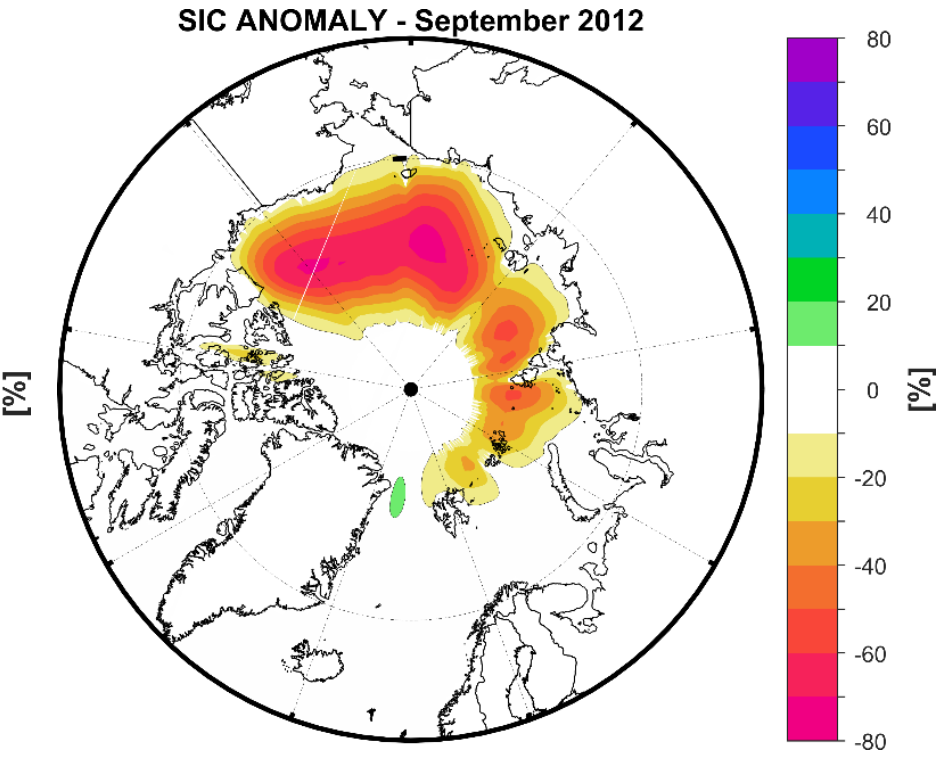

c)

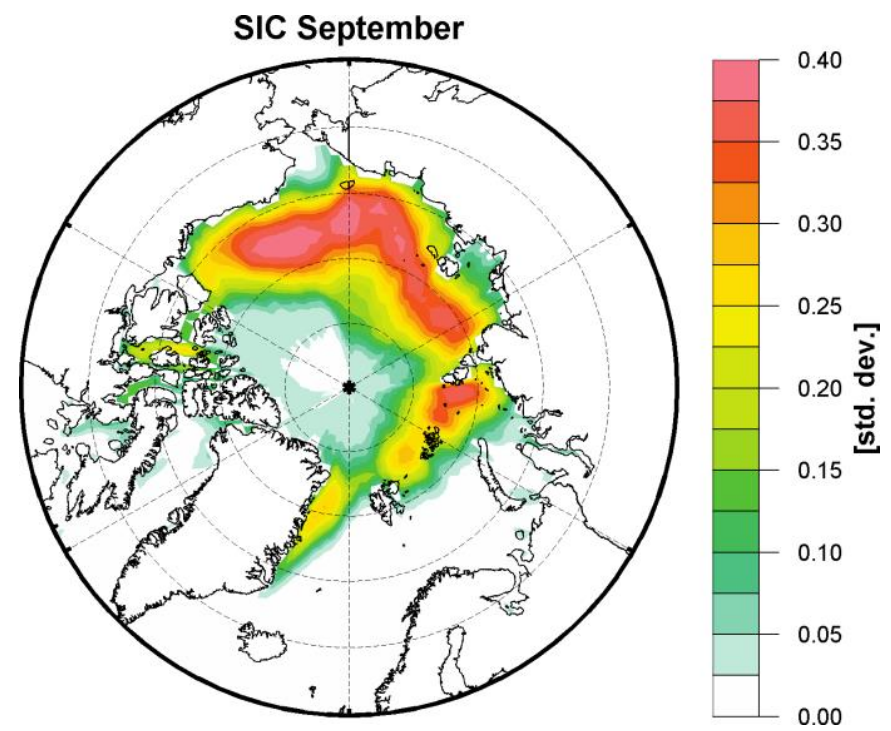

Figure S1. a) September sea ice concentration anomalies for 2007; b) as in a) but for 2012 and c) the standard deviation of the September sea ice concentration. 


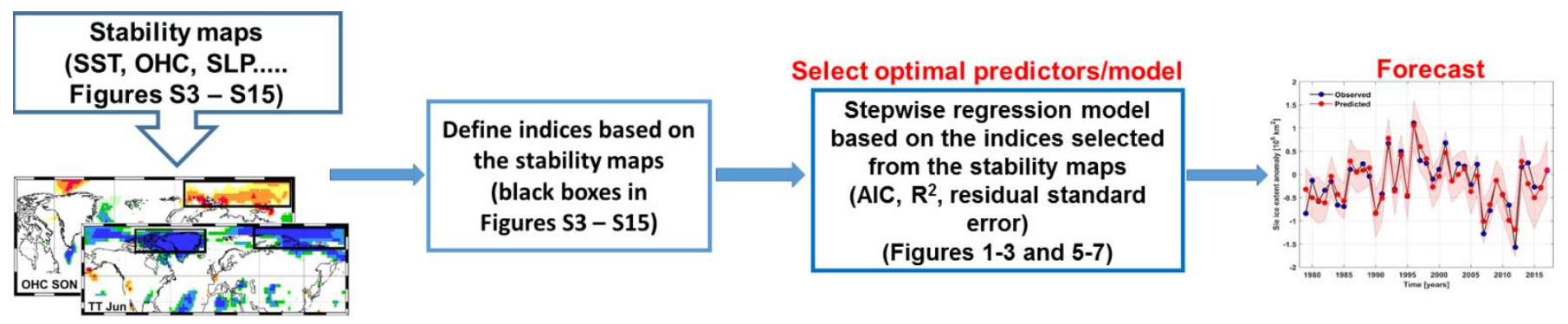

Figure S2. Workflow of the selection of the optimal predictors/model. 

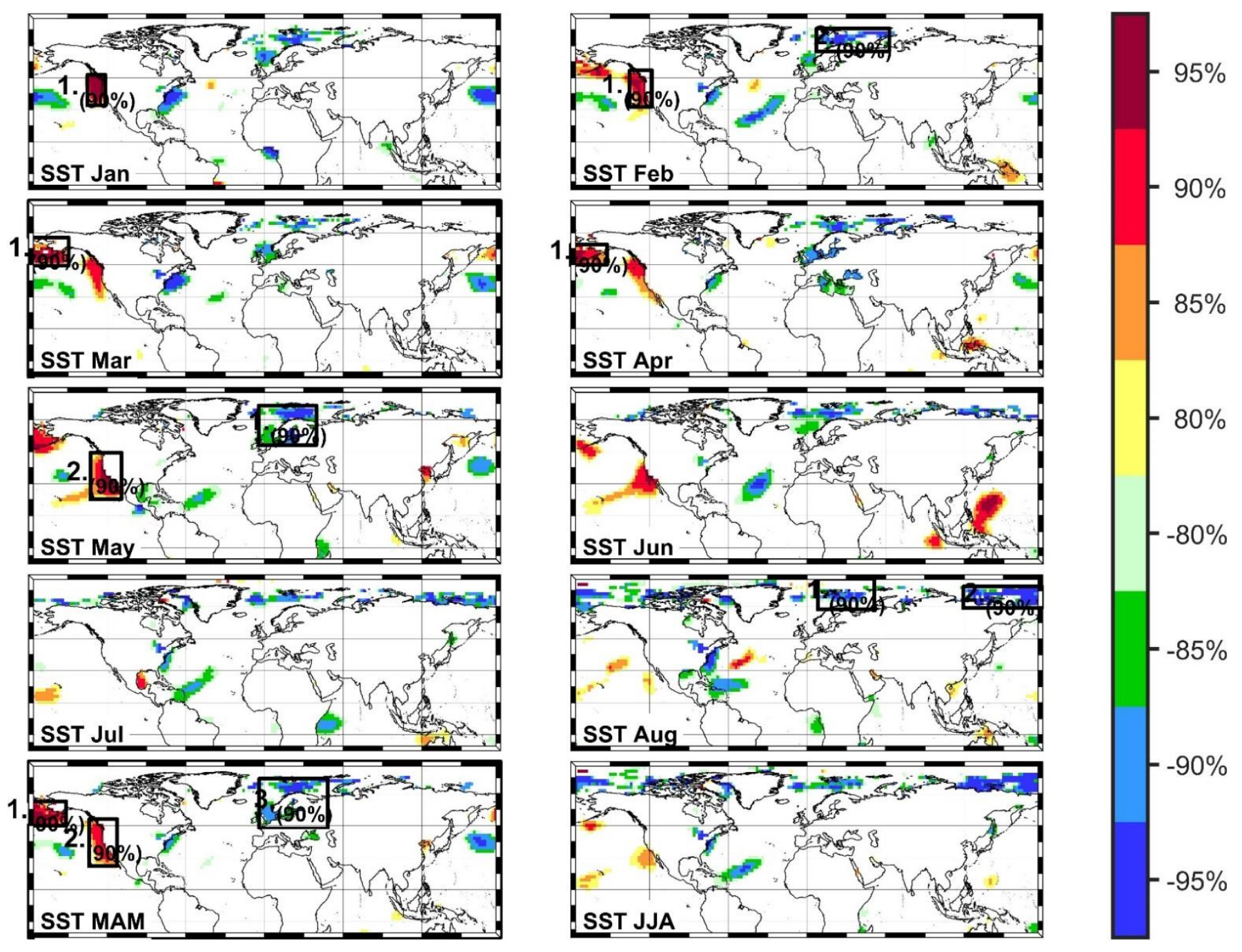

Figure S3. The stability map between September sea ice extent and sea surface temperature (SST) with different lags (SST leads the sea ice extent). The black boxes represent the areas used in the regression model. Regions where the correlation is stable, positive and significant for at least $80 \%$ of the 21 -year windows are shaded with dark red $(95 \%)$, red $(90 \%)$, orange $(85 \%)$ and yellow $(80 \%)$. The corresponding regions where the correlation is stable, but negative, are shaded with dark blue $(95 \%)$, blue $(90 \%)$, green $(85 \%)$ and light green $(80 \%)$. 

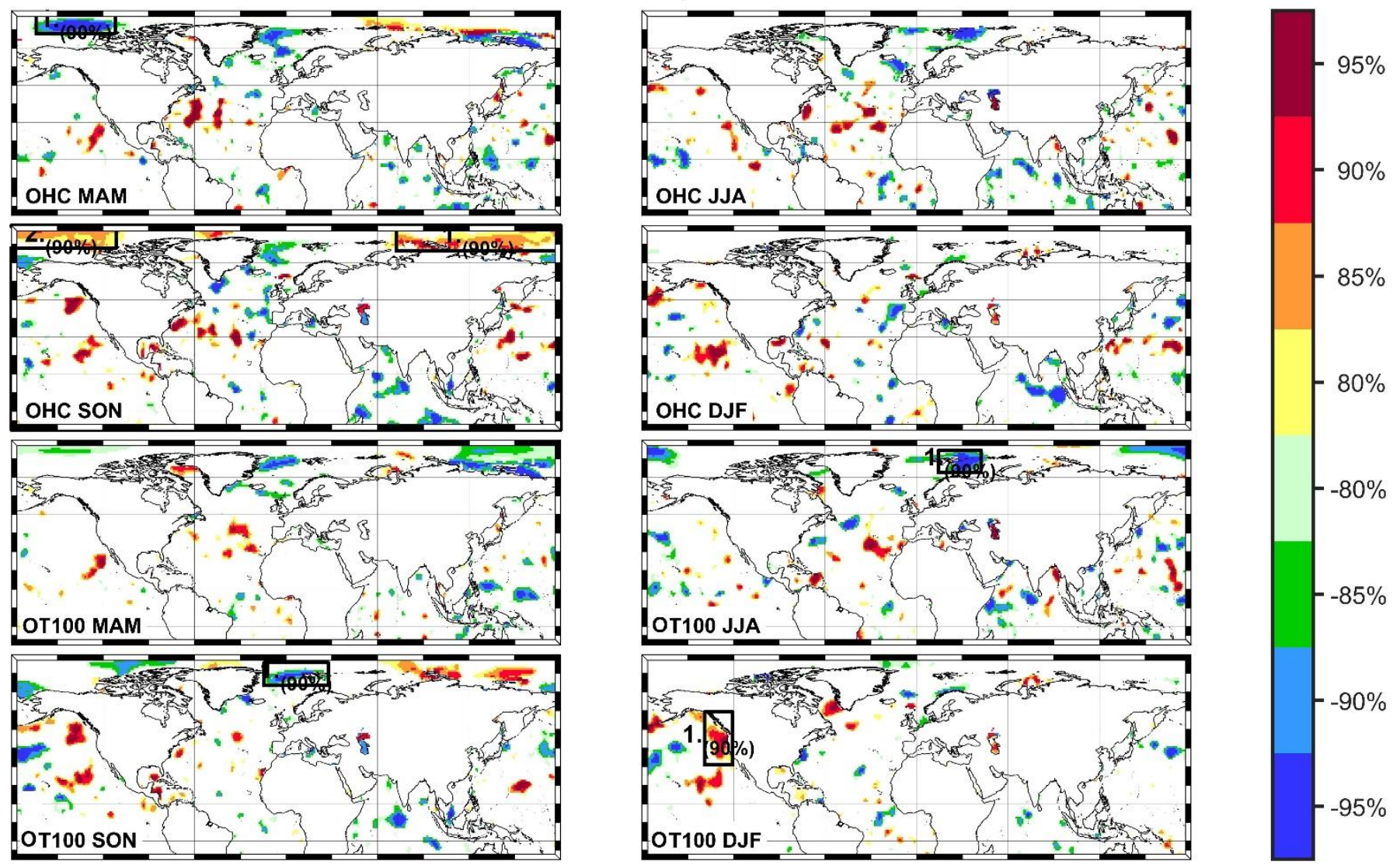

Figure S4. The stability map between September sea ice extent and the seasonal heat content in the first $700 \mathrm{~m}(\mathrm{OHC})$ and the seasonal upper ocean temperature in the first 100m (TT100) with different lags (OHC and TT100 leads the sea ice extent). The black boxes represent the areas used in the regression model. Regions where the correlation is stable, positive and significant for at least $80 \%$ of the 21 -year windows are shaded with dark red $(95 \%)$, red $(90 \%)$, orange $(85 \%)$ and yellow $(80 \%)$. The corresponding regions where the correlation is stable, but negative, are shaded with dark blue $(95 \%)$, blue $(90 \%)$, green $(85 \%)$ and light green $(80 \%)$. 

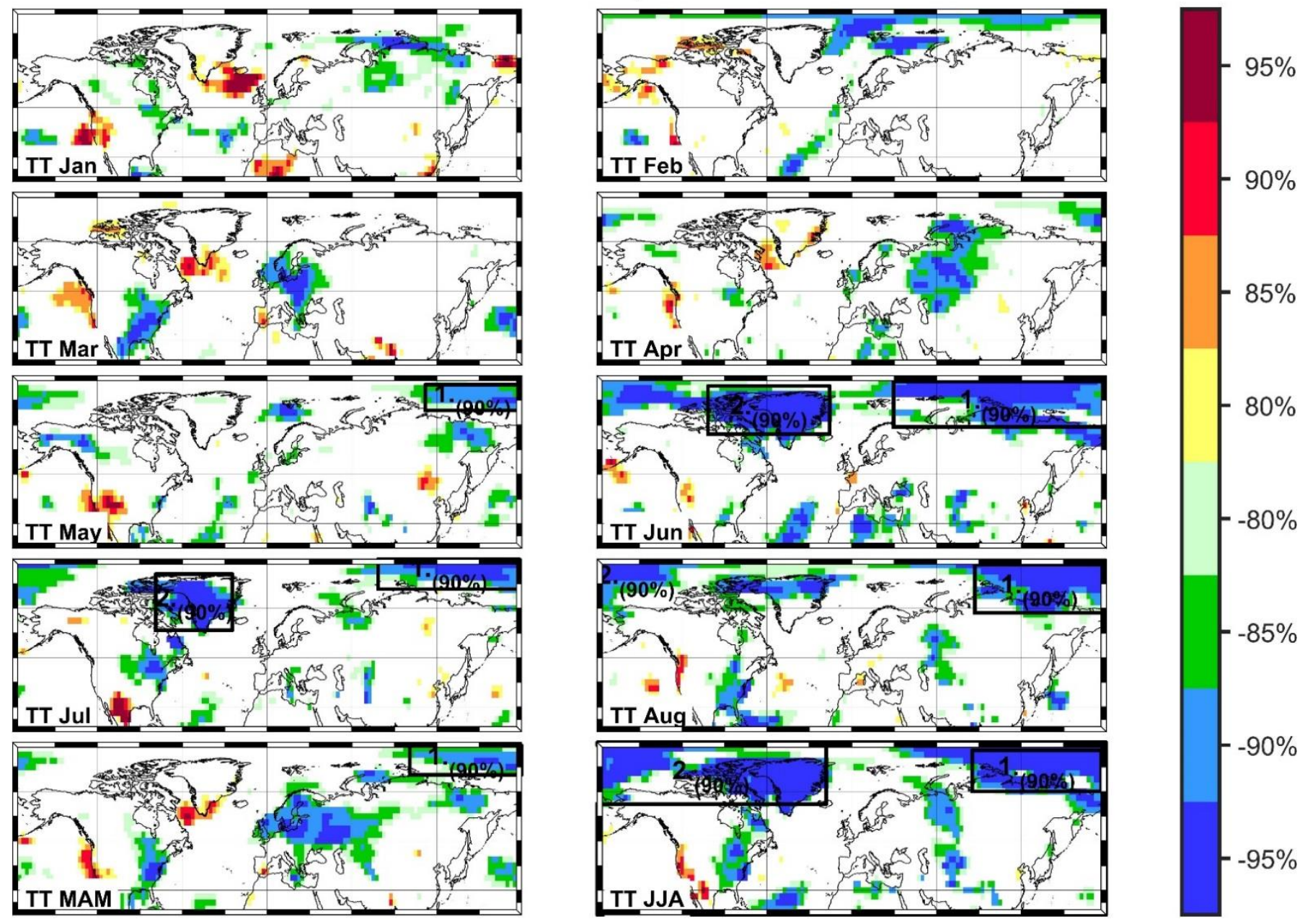

Figure S5. The stability map between September sea ice extent and air temperature (TT) with different lags (TT leads the sea ice extent). The black boxes represent the areas used in the regression model. Regions where the correlation is stable, positive and significant for at least $80 \%$ of the 21 -year windows are shaded with dark red $(95 \%)$, red $(90 \%)$, orange $(85 \%)$ and yellow $(80 \%)$. The corresponding regions where the correlation is stable, but negative, are shaded with dark blue (95\%), blue $(90 \%)$, green $(85 \%)$ and light green $(80 \%)$. 

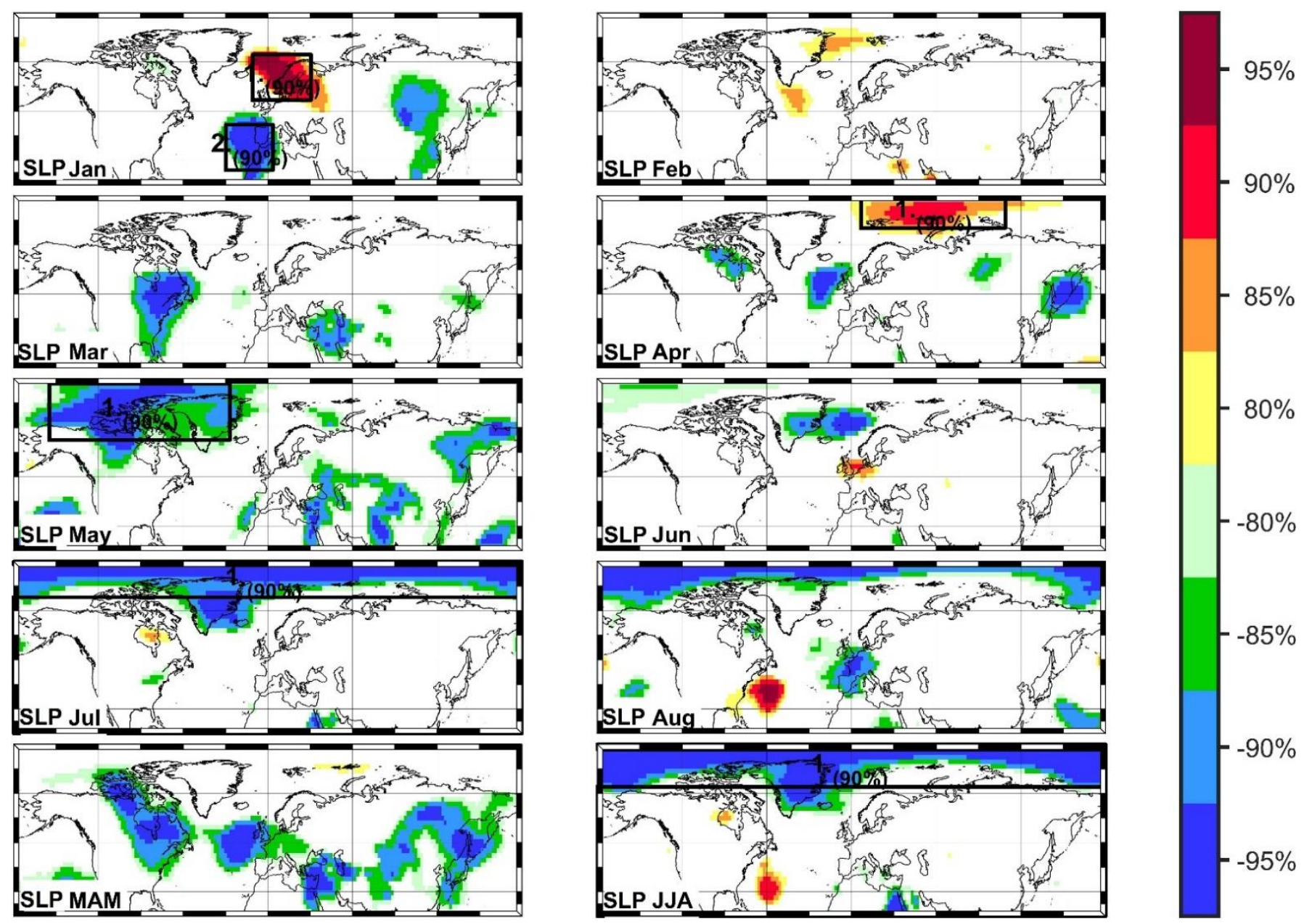

Figure S6. The stability map between September sea ice extent and the mean sea level pressure (SLP) with different lags (SLP leads the sea ice extent). The black boxes represent the areas used in the regression model. Regions where the correlation is stable, positive and significant for at least $80 \%$ of the 21 -year windows are shaded with dark red (95\%), red (90\%), orange $(85 \%)$ and yellow $(80 \%)$. The corresponding regions where the correlation is stable, but negative, are shaded with dark blue $(95 \%)$, blue $(90 \%)$, green $(85 \%)$ and light green $(80 \%)$. 

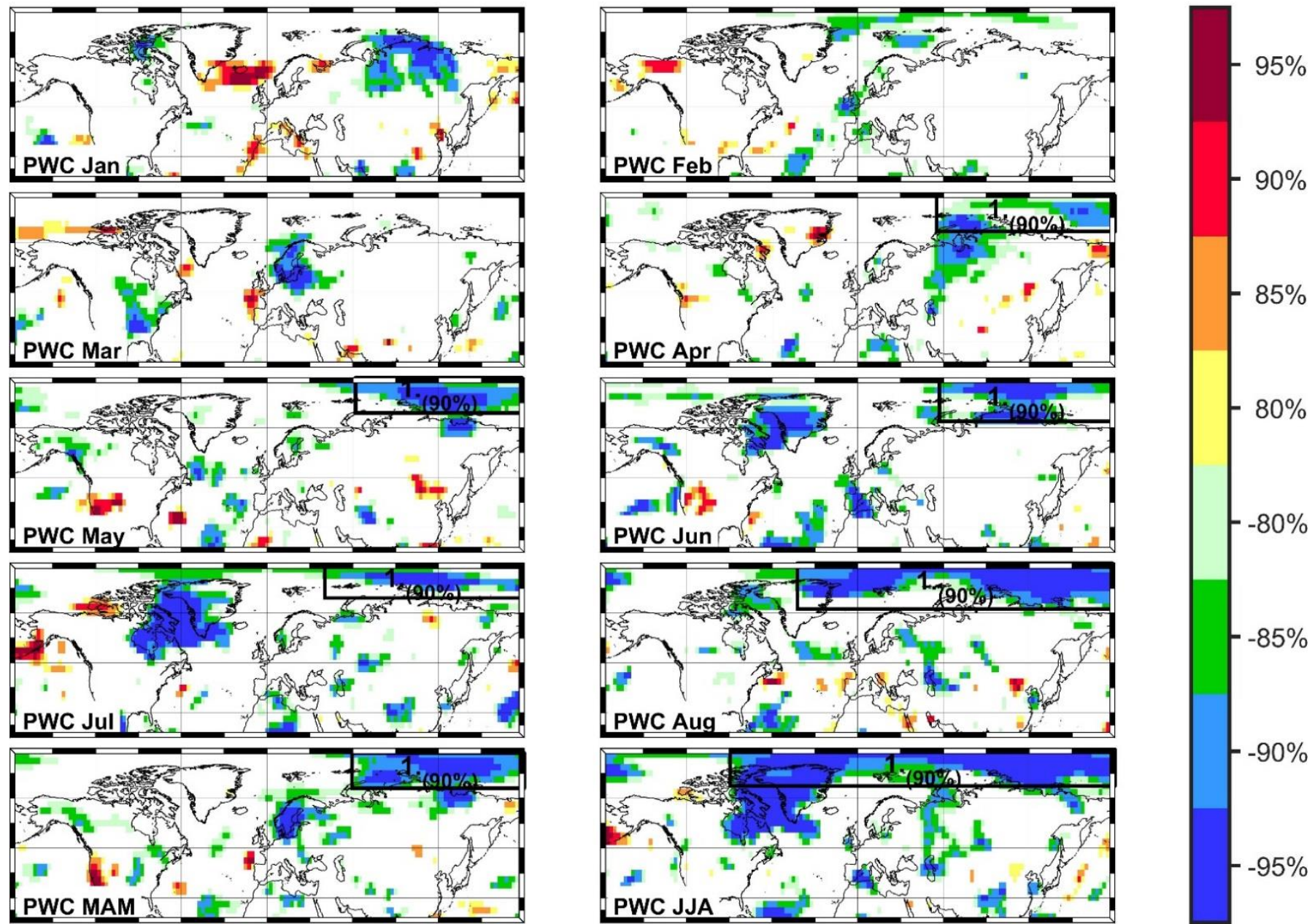

Figure S7. The stability map between September sea ice extent and the precipitable water content (PWC) with different lags (PWC leads the sea ice extent). The black boxes represent the areas used in the regression model. Regions where the correlation is stable, positive and significant for at least $80 \%$ of the 21 -year windows are shaded with dark red (95\%), red $(90 \%)$, orange $(85 \%)$ and yellow $(80 \%)$. The corresponding regions where the correlation is stable, but negative, are shaded with dark blue (95\%), blue (90\%), green $(85 \%)$ and light green $(80 \%)$. 

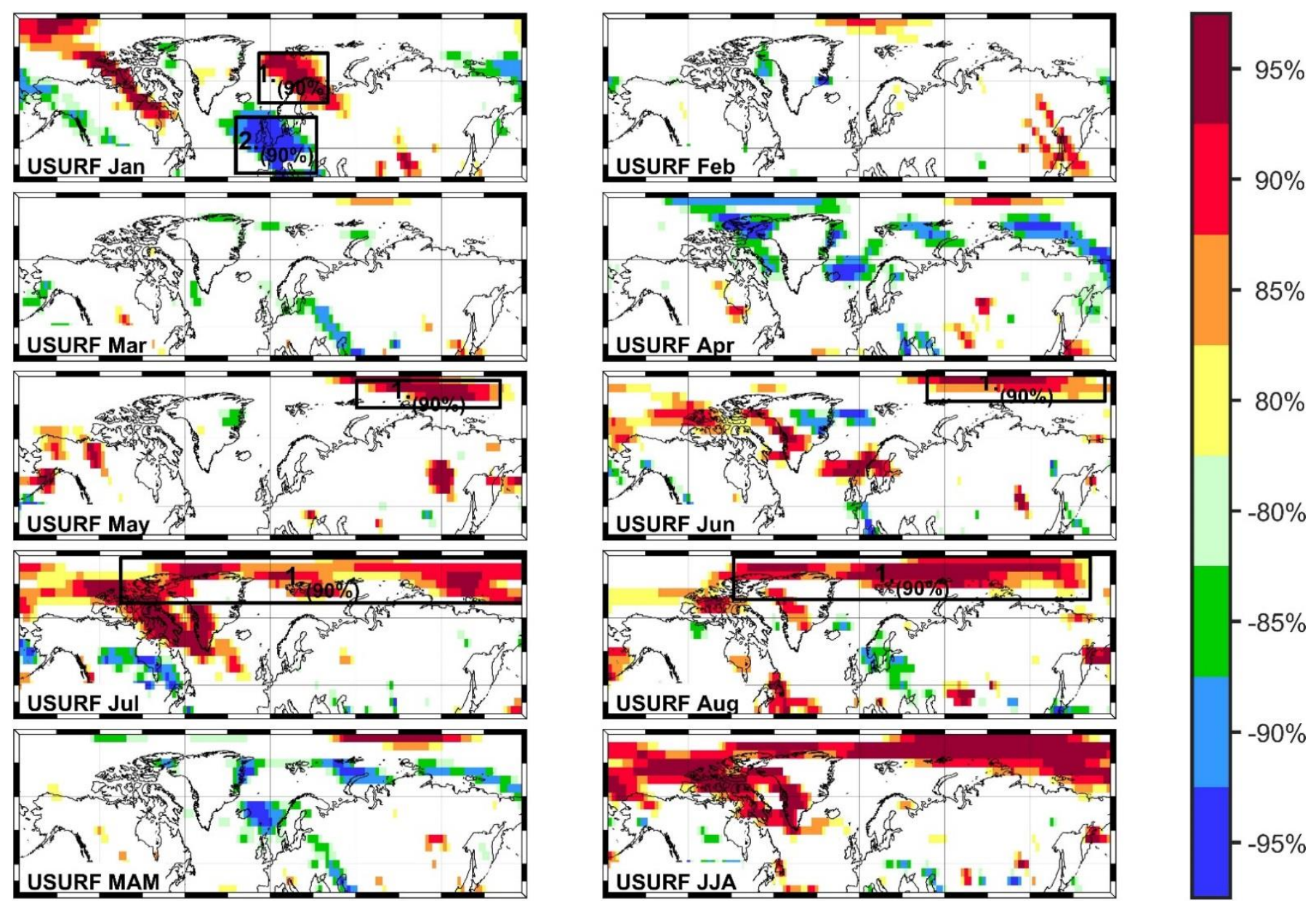

Figure S8. The stability map between September sea ice extent and the surface zonal wind (USURF) with different lags (USURF leads the sea ice extent). The black boxes represent the areas used in the regression model. Regions where the correlation is stable, positive and significant for at least $80 \%$ of the 21 -year windows are shaded with dark red (95\%), red $(90 \%)$, orange $(85 \%)$ and yellow $(80 \%)$. The corresponding regions where the correlation is stable, but negative, are shaded with dark blue (95\%), blue (90\%), green $(85 \%)$ and light green $(80 \%)$. 

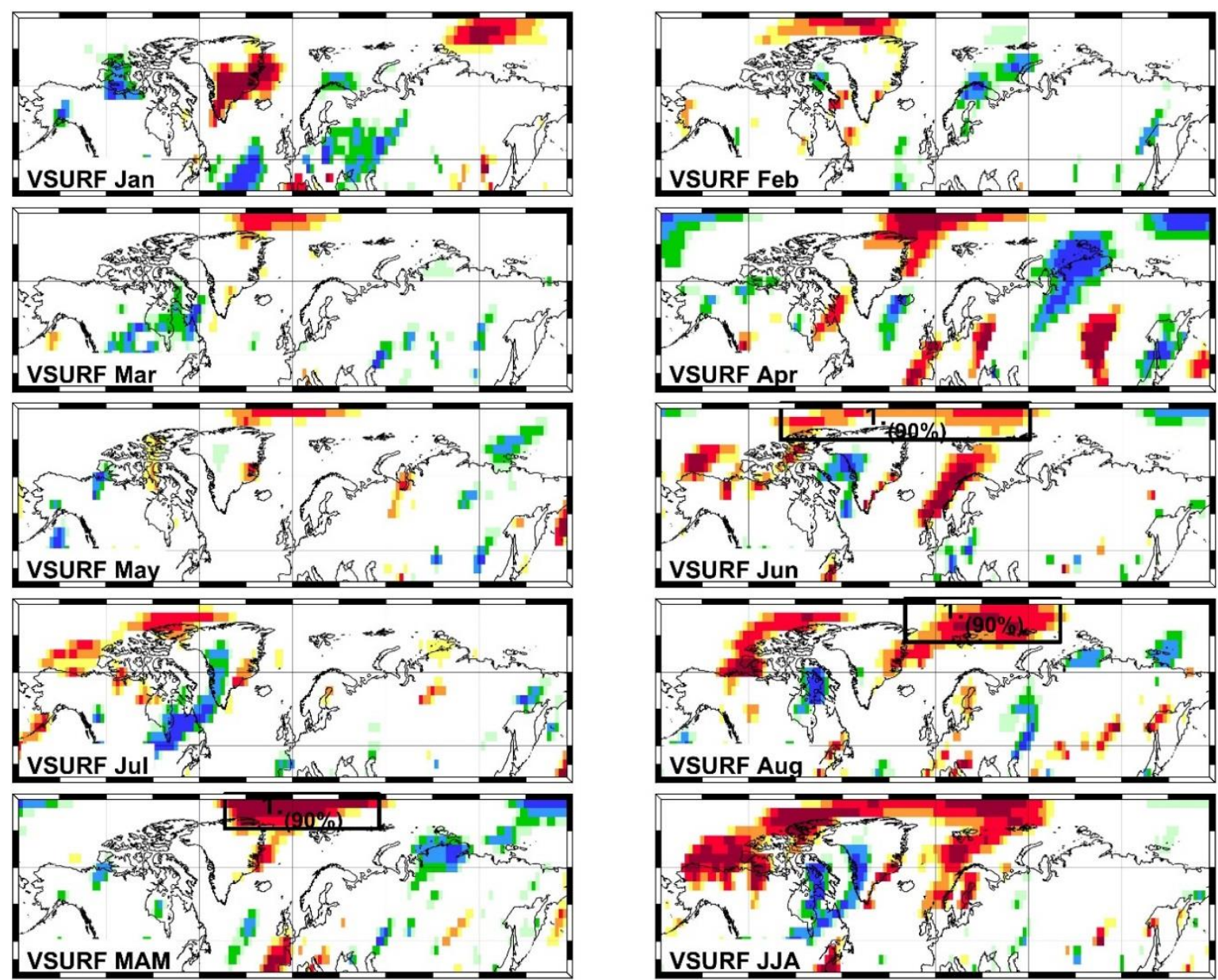

Figure S9. The stability map between September sea ice extent and surface meridional wind (VSURF) with different lags (VSURF leads the sea ice extent). The black boxes represent the areas used in the regression model. Regions where the correlation is stable, positive and significant for at least $80 \%$ of the 21-year windows are shaded with dark red (95\%), red $(90 \%)$, orange $(85 \%)$ and yellow $(80 \%)$. The corresponding regions where the correlation is stable, but negative, are shaded with dark blue (95\%), blue (90\%), green $(85 \%)$ and light green $(80 \%)$. 

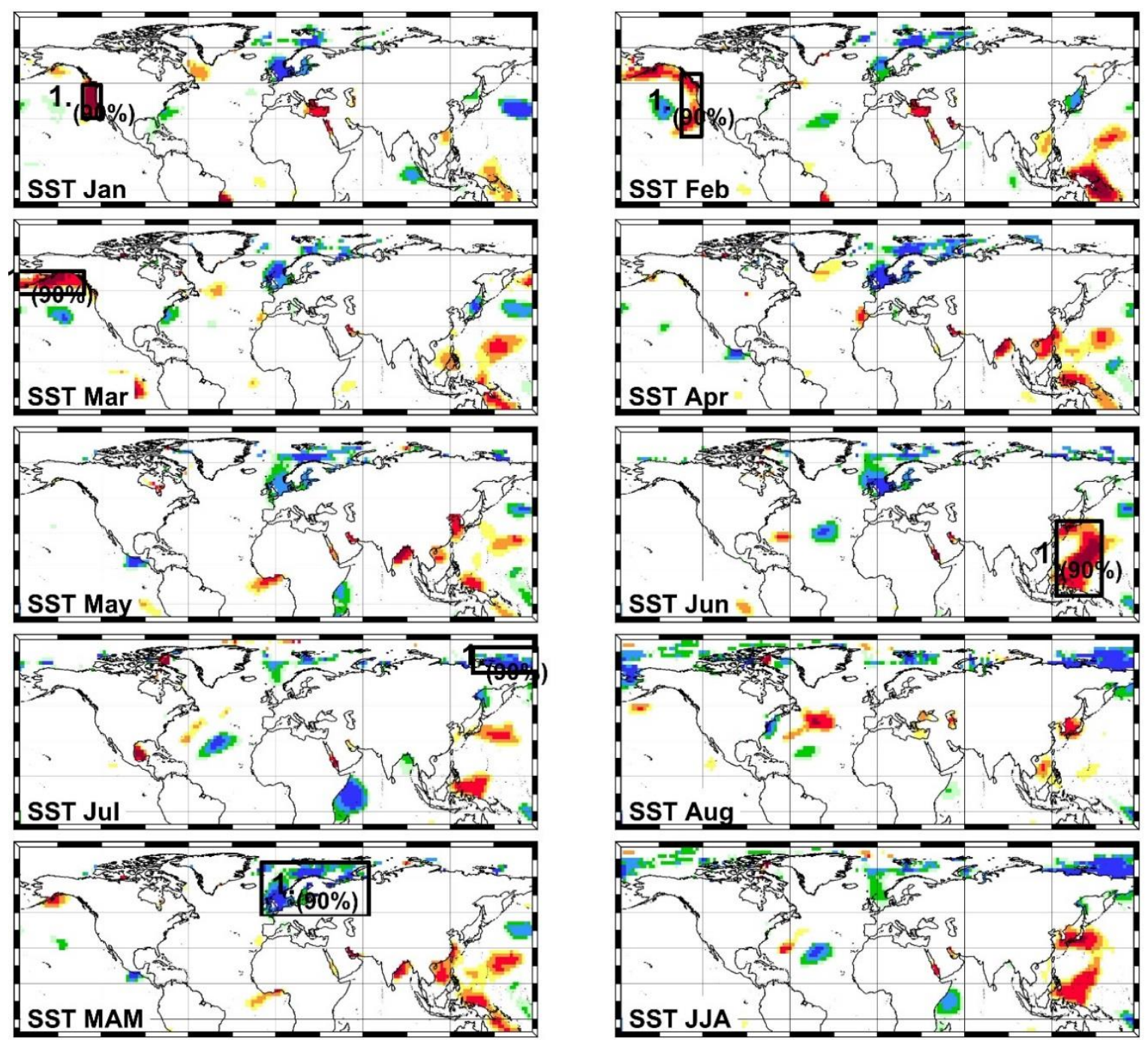

ISTT JJA

Figure S10. The stability map between September sea ice extent over the East Siberian Sea and sea surface temperature (SST) with different lags (SST leads the sea ice extent). The black boxes represent the areas used in the regression model. Regions where the correlation is stable, positive and significant for at least $80 \%$ of the 21-year windows are shaded with dark red $(95 \%)$, red $(90 \%)$, orange $(85 \%)$ and yellow $(80 \%)$. The corresponding regions where the correlation is stable, but negative, are shaded with dark blue $(95 \%)$, blue (90\%), green $(85 \%)$ and light green $(80 \%)$. 

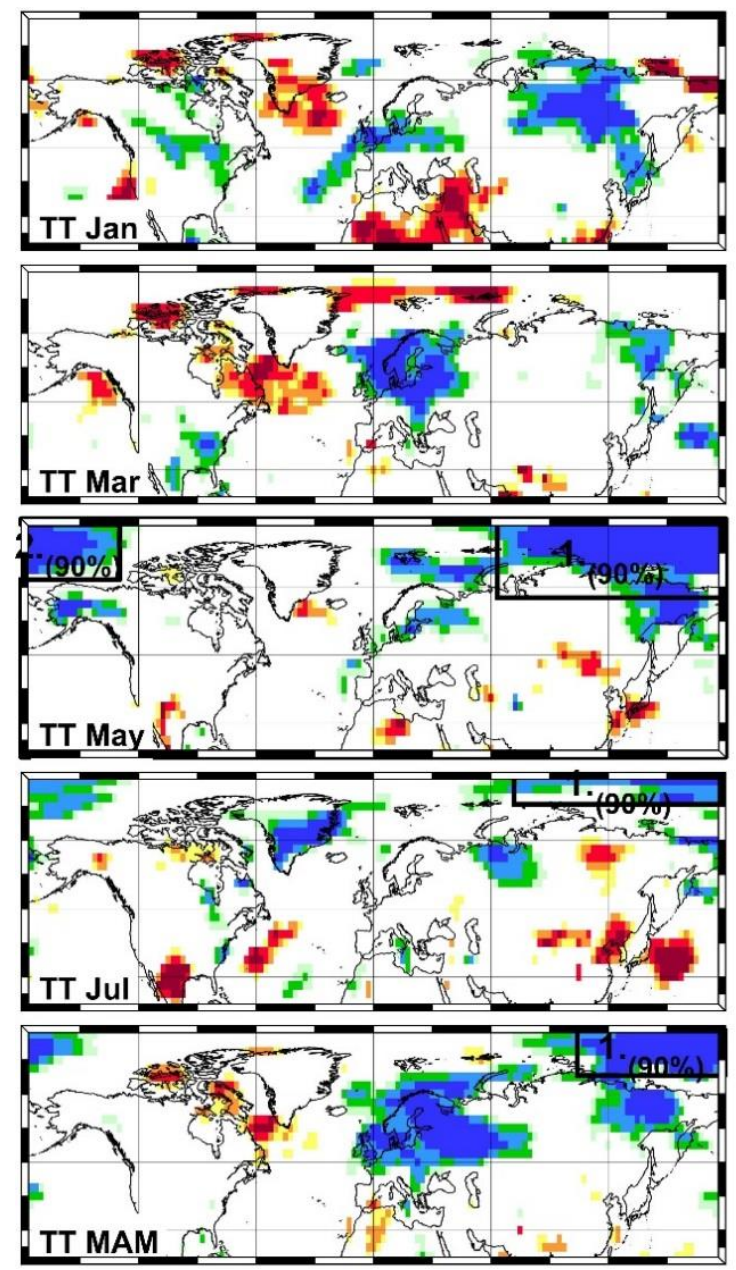

Figure S11. The stability map between September sea ice extent over the East Siberian Sea and air temperature (TT) with different lags (TT leads the sea ice extent). The black boxes represent the areas used in the regression model. Regions where the correlation is stable, positive and significant for at least $80 \%$ of the 21 -year windows are shaded with dark red (95\%), red $(90 \%)$, orange $(85 \%)$ and yellow $(80 \%)$. The corresponding regions where the correlation is stable, but negative, are shaded with dark blue $(95 \%)$, blue $(90 \%)$, green $(85 \%)$ and light green $(80 \%)$. 

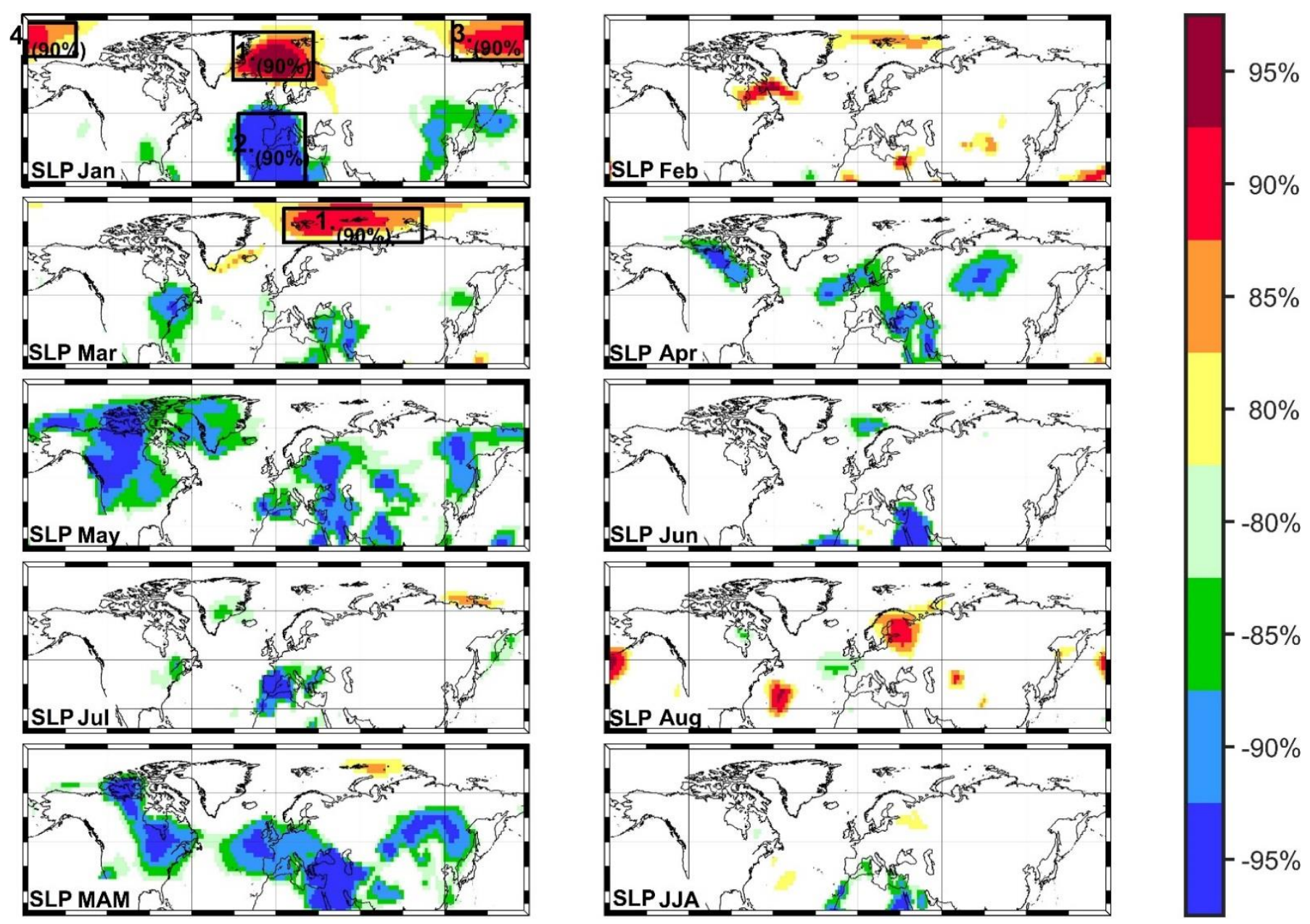

Figure S12. The stability map between September sea ice extent over the East Siberian Sea and the mean sea level pressure (SLP) with different lags (SLP leads the sea ice extent). The black boxes represent the areas used in the regression model. Regions where the correlation is stable, positive and significant for at least $80 \%$ of the 21 -year windows are shaded with dark red $(95 \%)$, red $(90 \%)$, orange $(85 \%)$ and yellow $(80 \%)$. The corresponding regions where the correlation is stable, but negative, are shaded with dark blue $(95 \%)$, blue $(90 \%)$, green $(85 \%)$ and light green $(80 \%)$. 

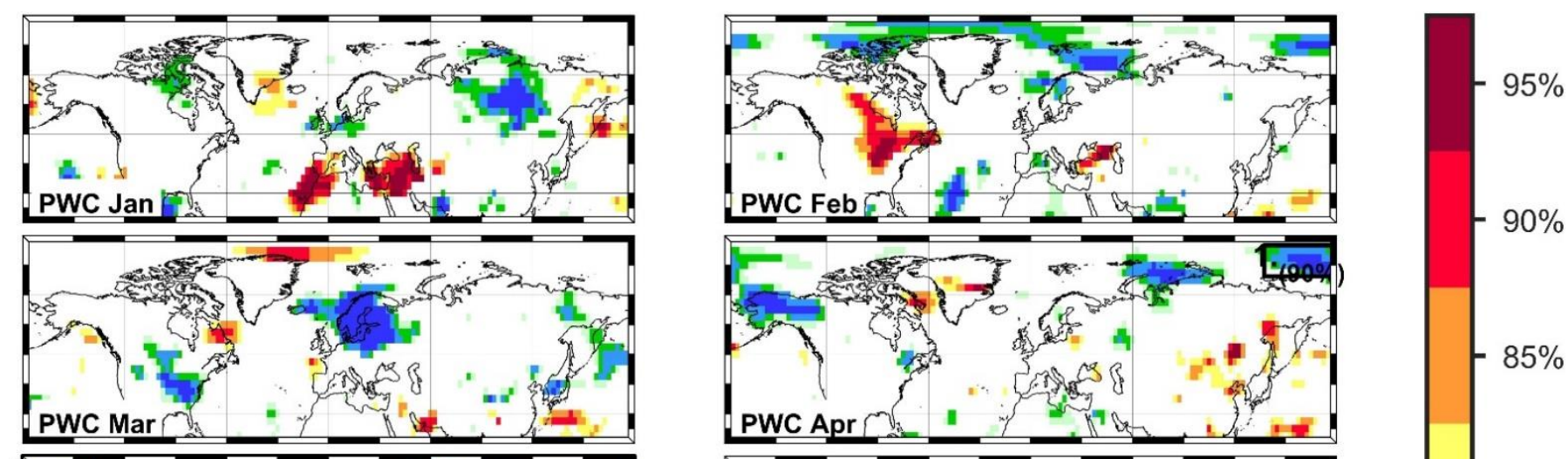

$90 \%$
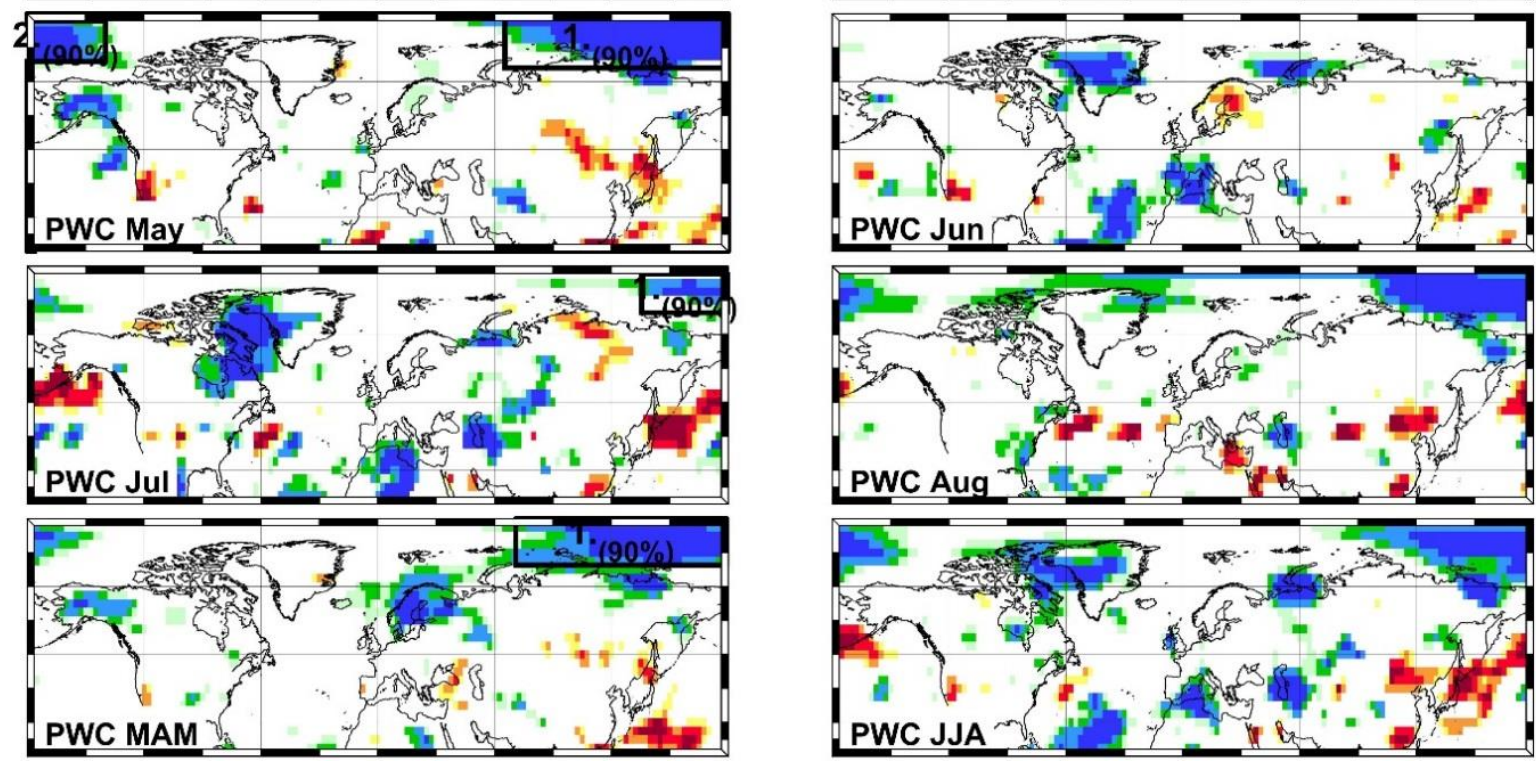

Figure S13. The stability map between September sea ice extent over the East Siberian Sea and the precipitable water content (PWC) with different lags (PWC leads the sea ice extent). The black boxes represent the areas used in the regression model. Regions where the correlation is stable, positive and significant for at least $80 \%$ of the 21 -year windows are shaded with dark red $(95 \%)$, red $(90 \%)$, orange $(85 \%)$ and yellow $(80 \%)$. The corresponding regions where the correlation is stable, but negative, are shaded with dark blue $(95 \%)$, blue $(90 \%)$, green $(85 \%)$ and light green $(80 \%)$. 


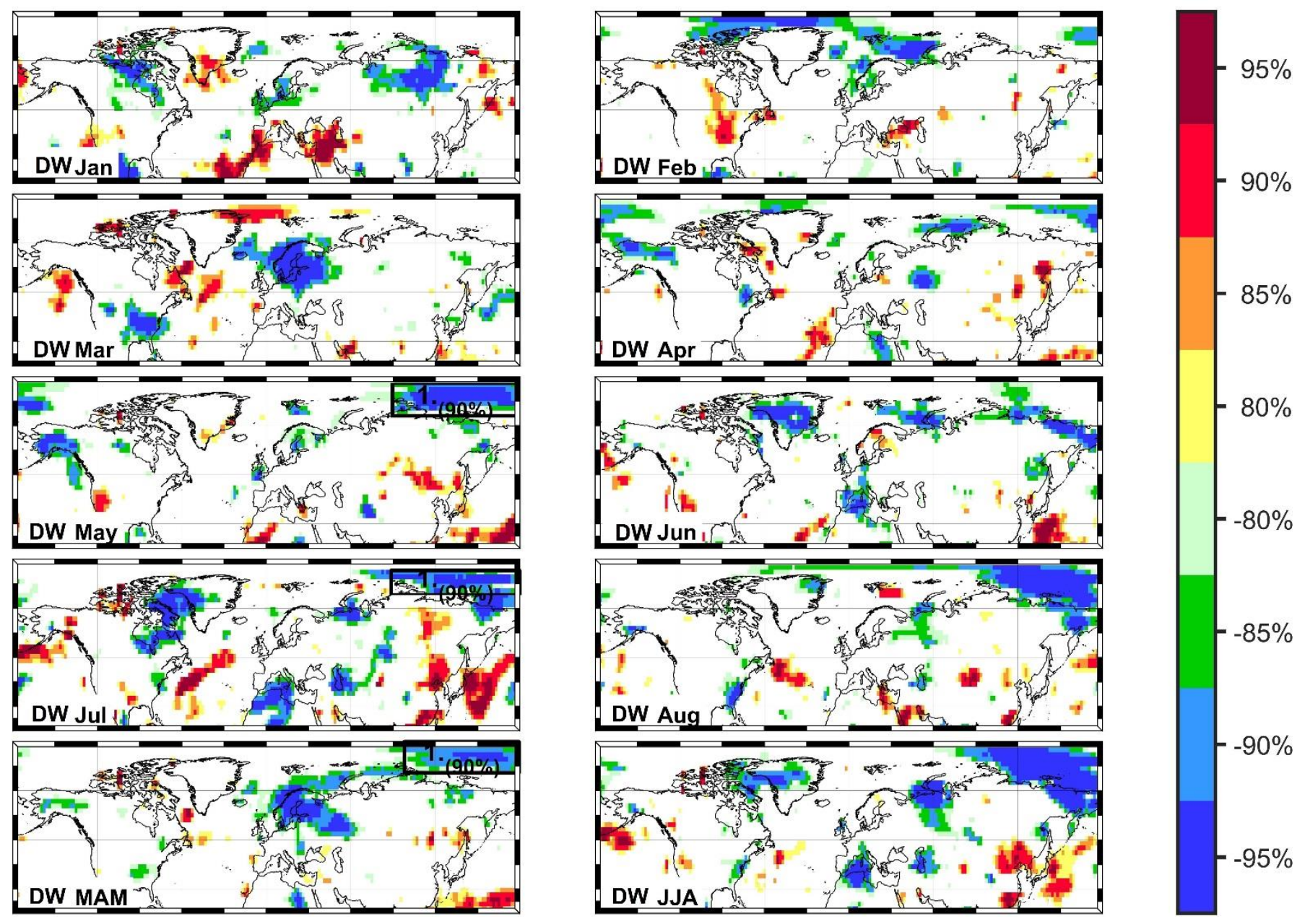

Figure S14. The stability map between September sea ice extent over the East Siberian Sea and the downward longwave radiation (DW) with different lags (DW leads the sea ice extent). The black boxes represent the areas used in the regression model. Regions where the correlation is stable, positive and significant for at least $80 \%$ of the 21 -year windows are shaded with dark red $(95 \%)$, red $(90 \%)$, orange $(85 \%)$ and yellow $(80 \%)$. The corresponding regions where the correlation is stable, but negative, are shaded with dark blue (95\%), blue (90\%), green (85\%) and light green $(80 \%)$. 

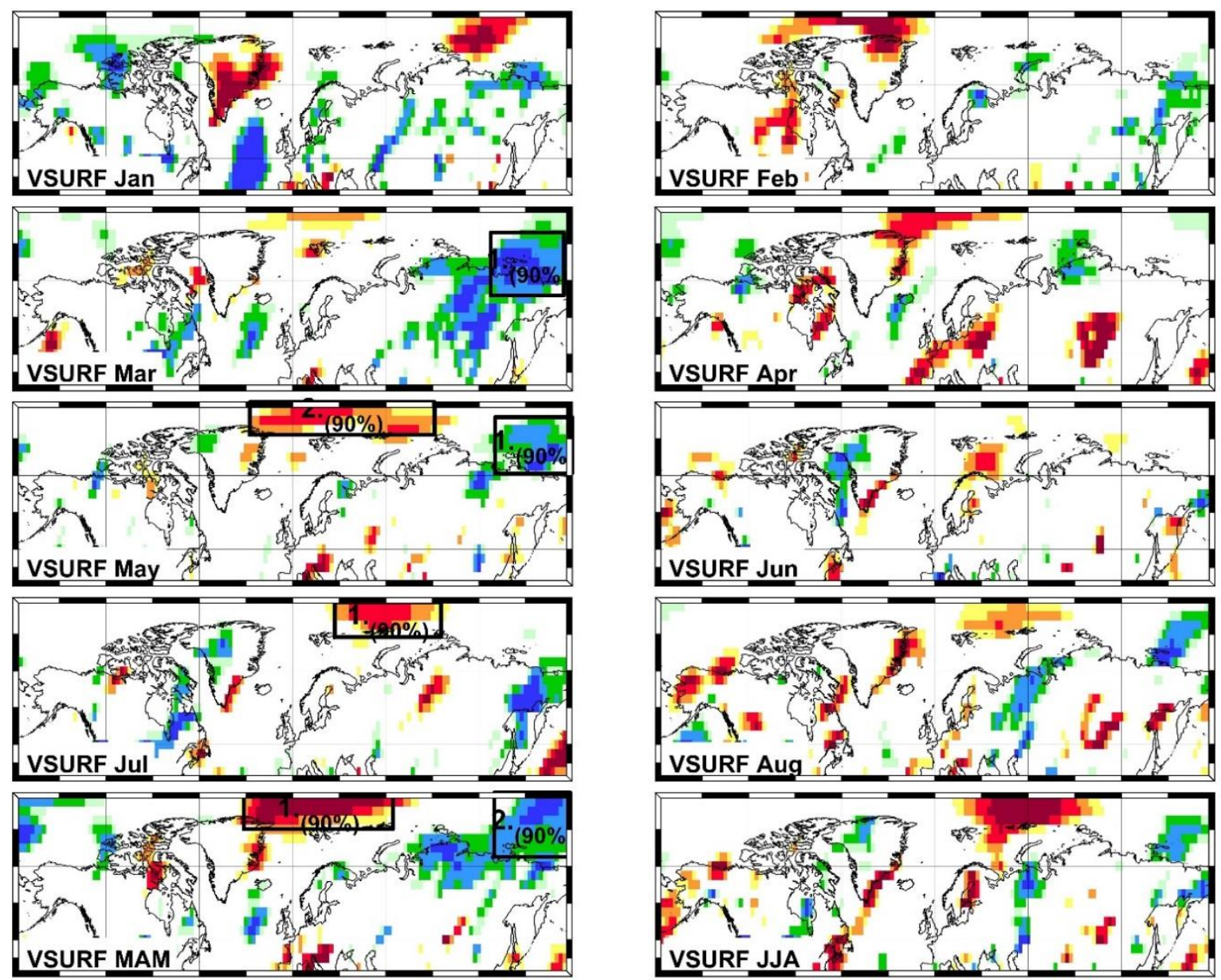

Figure S15. The stability map between September sea ice extent over the East Siberian Sea and the surface meridional wind (VSURF) with different lags (VSURF leads the sea ice extent). The black boxes represent the areas used in the regression model. Regions where the correlation is stable, positive and significant for at least $80 \%$ of the 21 -year windows are shaded with dark red $(95 \%)$, red $(90 \%)$, orange $(85 \%)$ and yellow $(80 \%)$. The corresponding regions where the correlation is stable, but negative, are shaded with dark blue (95\%), blue (90\%), green (85\%) and light green (80\%). 
Table S1. Skill parameters (see paragraph 2. Skill measures of for definition) based on different statistical methods for the observed and predicted pan-Arctic sea ice extent in September with different time lags.

\begin{tabular}{|l|r|r|r|r|r|r|}
\hline & \multicolumn{2}{|c|}{ May } & \multicolumn{2}{c|}{ June } & \multicolumn{2}{c|}{ July } \\
\hline & Calibration & \multicolumn{1}{|c|}{ Validation } & Calibration & \multicolumn{1}{c|}{ Validation } & Calibration & \multicolumn{1}{c|}{ Validation } \\
\hline MAE & 0.18 & 0.23 & 0.16 & 0.19 & 0.15 & 0.17 \\
\hline MSE & 0.05 & 0.08 & 0.04 & 0.06 & 0.04 & 0.05 \\
\hline RMSE & 0.22 & 0.29 & 0.2 & 0.24 & 0.19 & 0.23 \\
\hline NRMSE \% & 41.7 & 53.4 & 38 & 44.4 & 37.3 & 43 \\
\hline NSE & 0.82 & 0.68 & 0.85 & 0.78 & 0.86 & 0.8 \\
\hline mNSE & 0.54 & 0.42 & 0.6 & 0.53 & 0.62 & 0.58 \\
\hline rNSE & 0.94 & 0.42 & 0.96 & 0.68 & 0.96 & 0.75 \\
\hline d & 0.95 & 0.88 & 0.96 & 0.93 & 0.96 & 0.94 \\
\hline md & 0.76 & 0.66 & 0.79 & 0.74 & 0.81 & 0.78 \\
\hline rd & 0.98 & 0.78 & 0.99 & 0.9 & 0.99 & 0.92 \\
\hline cp & 0.91 & 0.85 & 0.94 & 0.89 & 0.94 & 0.89 \\
\hline r & 0.90 & 0.84 & 0.92 & 0.89 & 0.93 & 0.90 \\
\hline R & 0.81 & 0.71 & 0.85 & 0.79 & 0.86 & 0.81 \\
\hline
\end{tabular}


Table S2. Skill parameters (see paragraph 2. Skill measures of supplementary file for definition) based on different statistical methods for the observed and predicted East Siberian sea ice extent in September with different time lags.

\begin{tabular}{|l|r|r|r|r|r|r|}
\hline & \multicolumn{2}{|c|}{ May } & \multicolumn{2}{c|}{ June } & \multicolumn{2}{c|}{ July } \\
\hline & Calibration & \multicolumn{1}{|c|}{ Validation } & Calibration & Validation & Calibration & \multicolumn{1}{c|}{ Validation } \\
\hline MAE & 0.07 & 0.09 & 0.05 & 0.08 & 0.04 & 0.07 \\
\hline MSE & 0.01 & 0.01 & 0 & 0.01 & 0 & 0.01 \\
\hline RMSE & 0.08 & 0.12 & 0.07 & 0.1 & 0.06 & 0.09 \\
\hline NRMSE \% & 34.4 & 61.9 & 29.8 & 52.7 & 25 & 44.4 \\
\hline NSE & 0.88 & 0.57 & 0.91 & 0.69 & 0.94 & 0.78 \\
\hline mNSE & 0.61 & 0.33 & 0.69 & 0.43 & 0.75 & 0.44 \\
\hline rNSE & 1 & 0.83 & 1 & 0.87 & 0.98 & 0.9 \\
\hline d & 0.97 & 0.86 & 0.98 & 0.91 & 0.87 & 0.93 \\
\hline md & 0.81 & 0.65 & 0.84 & 0.71 & 0.71 \\
\hline rd & 1 & 0.94 & 1 & 0.96 & 0.94 & 0.97 \\
\hline cp & 0.88 & 0.81 & 0.91 & 0.86 & 0.97 & 0.90 \\
\hline r & 0.94 & 0.77 & 0.95 & 0.84 & 0.94 & 0.81 \\
\hline R & 0.88 & 0.58 & 0.91 & 0.71 & & 0.97 \\
\hline
\end{tabular}

